\title{
Cetuximab Rechallenge and Monotherapy in Patients with Squamous Cell Carcinoma of the Head and Neck
}

\section{Greg Knutzen Shanmuga Subbiah}

Arrowhead Regional Medical Center, Colton, Calif., and City of Hope Medical Group, Corona, Calif., USA

\section{Key Words}

Squamous cell carcinoma of the head and neck $\cdot$ Cetuximab $\cdot$ Rechallenge $\cdot$ Smoking

\begin{abstract}
Smokers with squamous cell carcinoma of the head and neck (SCCHN) have a particularly poor prognosis when compared with human papillomavirus-positive SCCHN. Here, we present case reports of two smokers with SCCHN treated with cetuximab-based therapy, highlighting the potential benefit of cetuximab before definitive chemoradiotherapy in patients with poor performance status as well as cetuximab rechallenge after progression. We conclude that cetuximab demonstrated notable effectiveness in two patients at high risk for poor prognosis, yielding a durable response in one and retaining activity on rechallenge in the other.

\section{Introduction}

Squamous cell carcinoma of the head and neck (SCCHN) is linked to alcohol and tobacco abuse [1] and, prior to the development of newer therapies, was associated with poor longterm survival [2]. Cetuximab, an anti-epidermal growth factor receptor monoclonal antibody, is associated with improved survival in locally advanced (LA) disease when added to radiotherapy (RT) compared with RT alone [3] and when added to first-line platinum/5fluorouracil (5-FU) in recurrent/metastatic (R/M) SCCHN [4]. Cetuximab is the only targeted agent currently approved in SCCHN; it is approved in LA SCCHN in combination with RT, in

KARGER125:s $12 \begin{aligned} & \text { Shanmuga Subbiah, MD, MRCP } \\ & \text { Arrowhead Regional Medical Center } \\ & 400 \text { N Pepper Avenue } \\ & \text { Colton, CA 92324 (USA) } \\ & \text { E-Mail Ssubbiah@coh.org }\end{aligned}$


Knutzen and Subbiah: Cetuximab Rechallenge and Monotherapy in Patients with Squamous Cell Carcinoma of the Head and Neck

R/M SCCHN as first-line therapy in combination with platinum/5-FU, and in platinumrefractory disease as monotherapy [5]. Here, we describe two cases of SCCHN treated with cetuximab-based therapy in a novel fashion.

\section{Case Report}

Patient 1 is a 50-year-old man with prior alcohol use and a smoking history of half a pack/day since age 7. Comorbidities include coronary artery disease, atherosclerotic heart disease, diabetes mellitus, hypertension, and hyperlipidemia. In July 2011, the patient presented with minor dysphagia, and a computed tomography (CT) scan revealed a right neck mass of $2.2 \times 2.9 \mathrm{~cm}$ that had been growing for 4 months (fig. 1a). A fine-needle aspiration sample was positive for squamous cell carcinoma (SCC). A CT scan of the chest, abdomen, and pelvis was negative. In late August, the patient was diagnosed with a stage III T0N2M0 SCCHN with no identifiable primary after extensive clinical and radiographic evaluation. Clinical examination before initiation of treatment indicated that the tumor had grown to 3.5 $\mathrm{cm}$ and identified a new $1.5-\mathrm{cm}$ nodal metastasis in the left neck. A positron emission tomography (PET) scan demonstrated hypermetabolic activity with a standardized uptake value of 3.9 in the left neck and 3.0 in the right neck node, respectively. In October 2011, the patient started cetuximab monotherapy due to poor performance status with a loading dose of 400 $\mathrm{mg} / \mathrm{m}^{2}$ followed by a weekly dose of $250 \mathrm{mg} / \mathrm{m}^{2}$, and a percutaneous endoscopic gastrostomy (PEG) tube was placed. Twenty-three days after initiation of cetuximab, an examination documented a decrease to $3 \mathrm{~cm}$ for the right neck mass and $1 \mathrm{~cm}$ for the left mass. With the patient's clinical status improving, at week 12 of cetuximab therapy, he started the first of 2 cycles of carboplatin area under the concentration curve (AUC) 5 on day 1 and capecitabine $625 \mathrm{mg} / \mathrm{m}^{2}$ twice daily on days 1-21. Four weeks after initiation of capecitabine, carboplatin, and cetuximab, a CT scan revealed the right mass as $1.8 \times 2 \mathrm{~cm}$ and the left mass as $<1 \mathrm{~cm}$ (fig. 1b). The patient began RT 6 days later and received a total of 6,480 cGy in 36 fractions over 12 weeks concurrent with cetuximab, which he completed in May 2012. Clinical examination and PET scan 12 weeks after completion of concurrent chemoradiation therapy revealed no evidence of disease. No evidence of recurrence was seen upon clinical examination in July 2013. In November 2013, the patient developed an enlarged nodule in the left side of his neck, and fine-needle aspiration confirmed metastatic SCC. Further, a PET scan performed in January 2014 indicated abnormal uptake in the anterior and superior aspects of the tongue but no discrete mass. In February 2014, he underwent left neck dissection, which revealed metastatic SCC in the left digastric lymph node and in level 2B lymph nodes. The patient began cetuximab monotherapy in February 2014 and continues with no progression as of June 2014.

Patient 2 is a 61-year-old former smoker who presented on October 2010 with a painful, necrotic, ulcerated tongue lesion and an 8-cm right neck mass that persisted for 9-10 months. A CT scan indicated a conglomerate nodal mass of $10 \times 6 \times 5 \mathrm{~cm}$, and biopsy of the soft palate identified keratinizing invasive SCC. In November 2010, the patient was diagnosed with stage IVA T4aN2bM0 tonsillar SCC. A PEG tube was placed, and the patient received 3 cycles of induction TPF (3-week cycles of docetaxel and cisplatin, both $75 \mathrm{mg} / \mathrm{m}^{2}$, on day 1 and a continuous infusion of 5 -FU $1,000 \mathrm{mg} / \mathrm{m}^{2} /$ day on days $1-4$ ) followed by chemoradiation (weekly carboplatin AUC 1.5 and intensity-modulated RT of 60 cGy to the upper neck and 5,040 cGy in 38 fractions to the lower neck over 9 weeks). The patient concluded locoregional therapy in March 2011, and a subsequent PET scan 4 months later revealed no abnormal uptake. However, 10 days later, a local recurrence that manifested as 
Knutzen and Subbiah: Cetuximab Rechallenge and Monotherapy in Patients with Squamous Cell Carcinoma of the Head and Neck

pain to the tongue was confirmed. The patient declined chemotherapy at that time. The disease progressed in the subsequent 5 months with worsening neck pain, significant weight loss, and metastasis to the lung as evidenced by the PET/CT scan done on December 2011 (fig. 2a). Cetuximab monotherapy with a $400 \mathrm{mg} / \mathrm{m}^{2}$ loading dose was started on January 19 , 2012 , followed by a $250 \mathrm{mg} / \mathrm{m}^{2}$ weekly dose. After 23 days, the patient's tongue pain had resolved, and a PET/CT scan done in May 2012 showed complete resolution of uptake in the lung (fig. 2b) and minimal uptake in the tongue. In September 2012, approximately 41 weeks after initiation of cetuximab, a PET/CT scan to the neck and chest revealed no evidence of metastases or disease recurrence (fig. 2c), and the patient subsequently discontinued cetuximab. However, recurrence was suspected approximately 3 months later after another CT scan to the neck and chest identified a $3.1 \times 2.2 \times 1.7$ mass at the base of the tongue and a soft-tissue nodule at the right apex of the tongue. Recurrence was confirmed via a tongue biopsy that indicated keratinizing SCC with extensive associated ulcerations. Based on the previous response, the patient was rechallenged with cetuximab in March 2013, after which a PET scan at 1 month showed markedly decreased hypermetabolic activity at the base of the tongue and normal uptake in the lung. In December 2013, he reported that the pain to the left side of his tongue had returned, and in January 2014, he reported a small ulceration to his tongue on the left side. The patient declined biopsy and further radiation to the area. He continued on cetuximab until March 2014 when progression of cancer on the superior aspect of the tongue was documented. Upon this progression, the patient stopped treatment with cetuximab and began treatment with capecitabine, with resulting resolution of tongue ulceration and decreasing mass on the tongue.

\section{Discussion}

Recent advances in therapy including intensity-modulated RT and cetuximab have improved outcomes in patients with SCCHN [6]. However, treatment of patients with SCCHN associated with alcohol or tobacco use remains challenging because these patients have worse clinical outcomes [7]. The two cases described here demonstrate the antitumor efficacy of cetuximab in patients who were heavy smokers.

Patient 1 initially received 12 weeks of cetuximab monotherapy, which had sufficient antitumor activity to induce shrinkage of the primary tumor and nodal metastasis and improved his performance status to receive more definitive therapy. The patient subsequently received 2 cycles of capecitabine and carboplatin with cetuximab, which contributed to further shrinkage of both the primary tumor and nodal lesion. Definitive cetuximab and RT were administered, after which a PET scan revealed no evidence of disease, and a durable remission was achieved.

Patient 2 initially received sequential therapy with induction TPF and definitive carboplatin/RT. After conclusion of treatment, the tumor recurred and metastasized to the lung. Both the recurrent tumor and lung metastasis completely responded to approximately 10 months of cetuximab monotherapy. Although another recurrence emerged 3 months later, retreatment with cetuximab appeared to retain activity because a subsequent PET scan indicated a reduction in hypermetabolic activity at the site of recurrence. This case demonstrates both the effectiveness of cetuximab in a heavily pretreated patient and the activity of cetuximab rechallenge after disease progression.

Although human papillomavirus (HPV) status was not used to guide treatment decisions, it was assessed retrospectively. The primary tumors of both patients were HPV negative. The efficacy of cetuximab monotherapy in patients with HPV-negative disease is sup- 
Knutzen and Subbiah: Cetuximab Rechallenge and Monotherapy in Patients with Squamous Cell Carcinoma of the Head and Neck

ported by the retrospective analysis of the EXTREME trial, which found that cetuximab plus platinum/fluorouracil was effective in R/M SCCHN regardless of HPV status [8].

Patient 2 had a positive response to cetuximab rechallenge. Few clinical studies have evaluated rechallenge with targeted therapies, and to date, there are no convincing predictive factors suggesting which drug should be readministered. Although there are no reports in the literature of cetuximab rechallenge in SCCHN, rechallenge with the same cetuximabbased chemotherapy improved progression-free survival in a phase 2 study in patients with colorectal cancer [9].

The outcomes observed in patients 1 and 2 show that cetuximab is effective and should be investigated in other SCCHN treatment settings. For example, given the activity of cetuximab monotherapy, maintenance cetuximab could be used to prevent tumor recurrence following definitive therapy in LA SCCHN. The role of rechallenge therapy in recurrent SCCHN is not defined but could be considered for fit patients who do not have other options. A randomized multicenter trial would be needed to confirm this observation prior to implementation in clinical practice.

In conclusion, cetuximab demonstrated notable effectiveness in two patients at high risk for poor prognosis, yielding a durable response in one and retaining activity on rechallenge in the other. Since cetuximab is a well-tolerated drug with minimal side effects, novel ways to use this drug as described here should be evaluated. Multicenter randomized trials in patients with SCCHN and poor performance status are needed to identify biomarkers predictive of response to cetuximab during rechallenge and in the neoadjuvant setting.

\section{Acknowledgements}

The authors acknowledge medical writing assistance provided by Clinical Thinking (Hamilton, N.J., USA), which was funded by Bristol-Myers Squibb.

\section{Statement of Ethics}

Written informed consent was obtained from each patient. Both the authors were involved in conception, design, data analysis, interpretation and manuscript writing of this article. Institutional ethics policy and patient privacy as per HIPPA guidelines were followed.

\section{Disclosure Statement}

The authors have no potential competing interests to declare.

\section{References}

1 Hashibe M, Brennan P, Chuang S, et al: Interaction between tobacco and alcohol use and the risk of head and neck cancer: pooled analysis in the International Head and Neck Cancer Epidemiology Consortium. Cancer Epidemiol Biomarkers Prev 2009;18:541-550.

-2 Pignon JP, Bourhis J, Domenge C, Designe L: Chemotherapy added to locoregional treatment for head and neck squamous-cell carcinoma: three meta-analyses of updated individual data. MACH-NC Collaborative Group. Meta-Analysis of Chemotherapy on Head and Neck Cancer. Lancet 2000;355:949-955. 


\section{Case Reports in Oncology}

\begin{tabular}{l|l}
\hline Case Rep Oncol 2015;8:503-508 \\
\hline DOI: 10.1159/000441019 & $\begin{array}{l}\text { ○ 2015 The Author(s). Published by S. Karger AG, Basel } \\
\text { www.karger.com/cro }\end{array}$ \\
\hline
\end{tabular}

Knutzen and Subbiah: Cetuximab Rechallenge and Monotherapy in Patients with Squamous Cell Carcinoma of the Head and Neck

-3 Bonner JA, Harari PM, Giralt J, et al: Radiotherapy plus cetuximab for locoregionally advanced head and neck cancer: 5-year survival data from a phase 3 randomised trial, and relation between cetuximab-induced rash and survival. Lancet Oncol 2010;11:21-28.

-4 Vermorken JB, Mesia R, Rivera F, et al: Platinum-based chemotherapy plus cetuximab in head and neck cancer. N Engl J Med 2008;359:1116-1127.

5 Bristol-Myers Squibb: Erbitux (Cetuximab) package insert, 2013.

-6 National Comprehensive Cancer Network. Head and Neck Cancers. NCCN Clinical Practice Guidelines in Oncology. Version 2.2014.

7 Ang KK, Harris J, Wheeler R, et al: Human papillomavirus and survival of patients with oropharyngeal cancer. N Engl J Med 2010;363:24-35.

8 Vermorken JB, Psyrri A, Mesia R, et al: Impact of tumor HPV status on outcome in patients with recurrent and/or metastatic squamous cell carcinoma of the head and neck receiving chemotherapy with or without cetuximab: retrospective analysis of the phase III EXTREME trial. Ann Oncol 2014;25:801-807.

-9 Santini D, Vincenzi B, Addeo R, et al: Cetuximab rechallenge in metastatic colorectal cancer patients: how to come away from acquired resistance? Ann Oncol 2012;23:2313-2318.
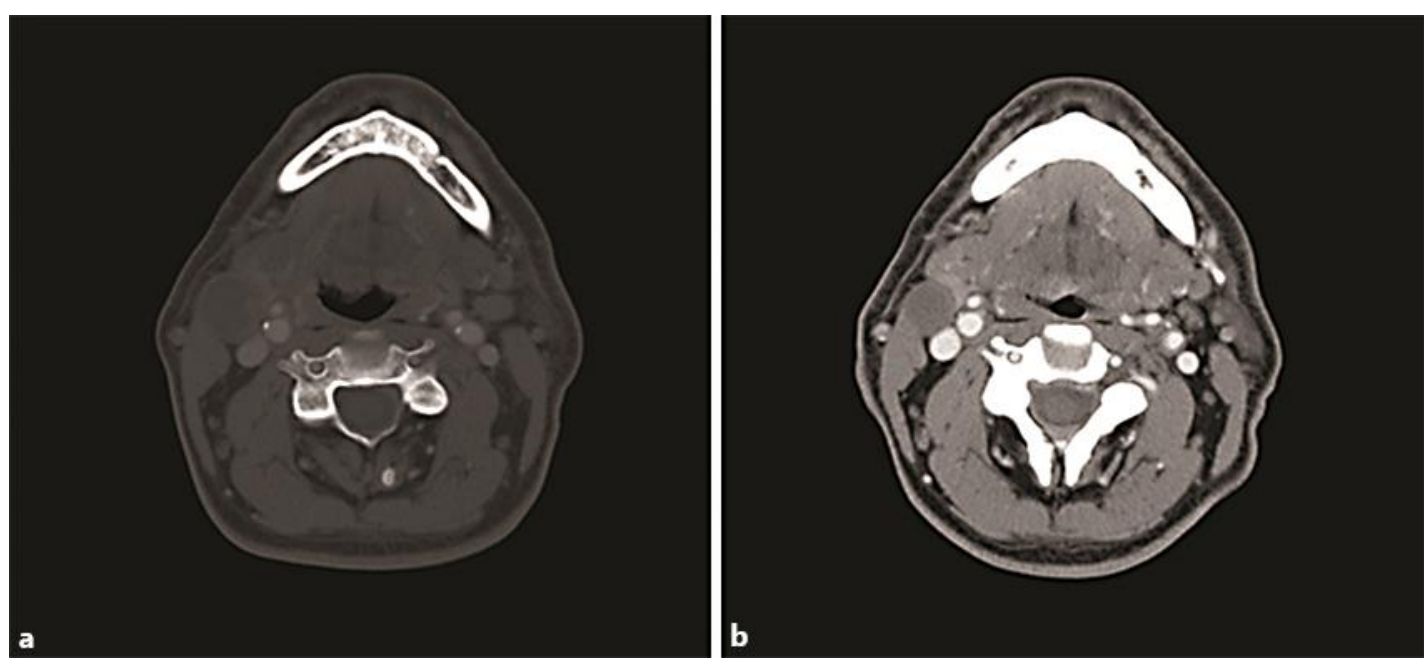

Fig. 1. Prediagnosis and on-treatment CT scans from patient 1 . a July 2011, before diagnosis: right mass of $2.2 \times 2.9 \mathrm{~cm}$ identified. b February 2012, after cetuximab, carboplatin, and capecitabine treatment: right mass of $1.8 \times 2 \mathrm{~cm}$ and left mass of $<1 \mathrm{~cm}$. 


\section{Case Reports in Oncology}

\begin{tabular}{l|l}
\hline Case Rep Oncol 2015;8:503-508 \\
\hline DOI: 10.1159/000441019 & $\begin{array}{l}\text { (c) 2015 The Author(s). Published by S. Karger AG, Basel } \\
\text { www.karger.com/cro }\end{array}$ \\
\hline
\end{tabular}

Knutzen and Subbiah: Cetuximab Rechallenge and Monotherapy in Patients with Squamous Cell Carcinoma of the Head and Neck
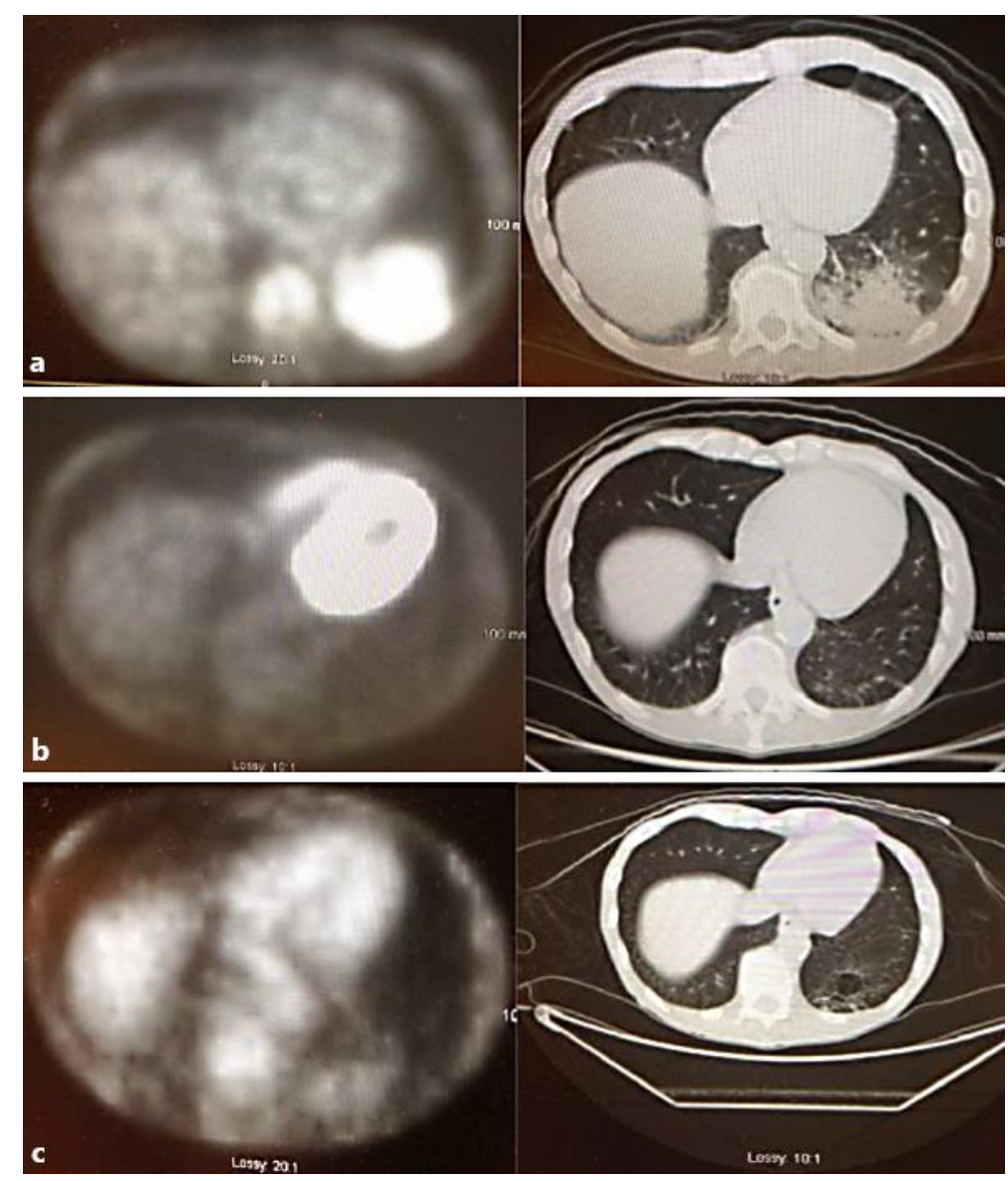

Fig. 2. Pretreatment and on-treatment PET scans from patient 2. a December 2011, after first recurrence, before cetuximab treatment: evidence of metastasis to the lung. b May 2012, approximately 4 months of cetuximab monotherapy: resolution of uptake in the lung. c September 2012: no evidence of metastases or disease recurrence. 